\title{
Prolongamento do Intervalo QT do Eletrocardiograma em Pacientes Reumáticos usando Antimaláricos ${ }^{(*)}$
}

\section{ECG QT Interval Prolongation in Rheumatic Patients using Antimalarial Druss}

\author{
Ludmilla Daru Rey ${ }^{(1)}$, Anderson Berneck ${ }^{(1)}$, Leonardo Gonçalves ${ }^{(1)}$, \\ Marilia B. Silva (2), Thelma L. Skare ${ }^{(2)}$ e José Antônio Silva ${ }^{(3)}$
}

\section{RESUMO}

Objetivo: estudar a influência de medicamentos antimaláricos (cloroquina e hidroxicloroquina) sobre o intervalo QTc do eletrocardiograma. Métodos: foram estudados 46 pacientes reumáticos em uso de antimaláricos, sendo 42 deles usuários de cloroquina e 4 de hidroxicloroquina. Todos faziam uso das doses habituais do medicamento $(7 \mathrm{mgkg} / \mathrm{dia}$ de hidroxicloroquina e 4 $\mathrm{mg} / \mathrm{kg} /$ dia de cloroquina), com exceção dos portadores de osteoartrite $(\mathrm{n}=8)$, os quais usavam metade desta dose. Os pacientes foram submetidos à ECG com medida do intervalo QTc, sendo considerado o valor normal máximo de 0,44 s para mulheres e 0,424 s para homens. Aqueles com resultado alterado eram convidados a retirar o medicamento e repetir o ECG para uma nova medida em duas semanas. Resultados: encontrou-se uma prevalência de $17,39 \%$ de aumento do intervalo QTc, o qual não estava relacionado com dose $(\mathrm{p}=0,574)$, tipo de antimalárico $(\mathrm{p}$ $=0,452)$ ou tempo de uso da droga $(p=0,09)$. Todos os pacientes, exceto um, concordaram em repetir o ECG, sendo verificada a reversão da anormalidade em todos. Conclusões: pacientes em uso de cloroquina podem apresentar aumento do intervalo QTc $(17,39 \%)$ e tal reação tem características idiossincráticas.

Palavras-chave: antimaláricos, cloroquina, hidroxicloroquina, QT longo, artrite.

\section{INTRODUÇÃO}

Os antimaláricos (cloroquina e hidroxicloroquina) fazem parte do armamentário terapêutico do reumatologista e são drogas amplamente utilizadas não só pela facilidade

\section{ABSTRACT}

Objective: to verify the influence of antimalarial drugs (chloroquine and hydroxychloroquine) on the QT interval of the electrocardiogram. Methods: we studied 46 rheumatic patients (42 using chloroquine and 4 using hydroxychloroquine), both taking the usual doses of such drugs $(7 \mathrm{mg} / \mathrm{kg} /$ day of hydroxychloroquine and $4 \mathrm{mg} / \mathrm{kg} /$ day of chloroquine) except for those with osteoarthritis $(n=8)$ who used half of the dose. Electrocardiogram was performed in all patients with measurements of QTC interval. Patients with abnormal results were advised to withdraw the medication and repeat the ECG within two weeks. Results: QTC interval prolongation was observed in $17.39 \%$ of the patients. Such abnormalities were not related to dose $(p=0.574)$, type of antimalarial drug ( $p$ $=0.452)$ and duration of drug use $(p=0.09)$. All patients with abnormal results, except for one, repeated the ECG with return to normal values. Conclusions: we concluded that patients taking chloroquine may have QTc prolongation as an adverse effect.

Keywords: antimalarial drugs, chloroquine, hydroxychloroquine, long QT, arthritis.

de administração como pelo seu baixo custo. Sendo drogas imunomoduladoras e antiinflamatórias, estão indicadas no tratamento da artrite reumatóide, do lúpus eritematoso sistêmico, da osteoartrite, da síndrome de Sjögren ${ }^{(1)}$ e da síndrome do anticorpo antifosfolípide ${ }^{(2)}$.

\footnotetext{
* Curso de Medicina da Faculdade Evangélica do Paraná. Recebido em 21/08/02. Aprovado, após revisão, em 28/02/03.

1. Médico(a) Residente do Serviço de Clínica Médica do Hospital Universitário Evangélico de Curitiba, PR.

2. Professora Assistente de Reumatologia do Curso de Medicina da Faculdade Evangélica do PR.

3. Médico Cardiologista do Hospital do Cajuru, Curitiba, PR. 
Essas drogas são extraídas da casca da cinchona e foram inicialmente usadas como antipiréticas pelos padres jesuítas, em $1650^{(3)}$. São medicamentos bem absorvidos pelo trato gastrintestinal, mesmo em presença de diarréia, e a cloroquina se liga mais extensamente a proteínas plasmáticas que a hidroxicloroquina ${ }^{(3)}$. Em casos de uso diário regular, essa primeira droga alcança equilíbrio de níveis plasmáticos em quatro a seis semanas, enquanto a hidroxicloroquina leva até seis meses para atingir o ponto de equilíbrio ${ }^{(3)}$. Ambas depositam-se extensamente em tecidos pigmentados, fígado, rim, pulmões e células sangüíneas ${ }^{(3)}$.

Como esperado, toda droga alberga um potencial de efeitos colaterais. Os efeitos colaterais mais temidos dos antimaláricos são os oftalmológicos e merecem, por parte dos médicos que se utilizam cronicamente deste medicamento, um processo de acompanhamento todo especial $^{(1)}$. Entretanto, estes não são únicos. Outros paraefeitos menos valorizados podem trazer repercussões que nem sempre são devidamente valorizadas por serem menos conhecidas.

Os antimaláricos têm repercussões no aparelho cardiovascular que têm sido descritas principalmente na forma de cardiomiotoxicidade e de bloqueios atrioventriculares ${ }^{(4-7)}$. Estas drogas sendo quimicamente similares à quinidina albergam o mesmo potencial que esta de provocar alterações no intervalo QT do eletrocardiograma $(\mathrm{ECG})^{(8)}$. Esta possibilidade, embora citada esparsamente na literatura, não é considerada pelos maiores usuários deste grupo de medicamentos que são os reumatologistas e os infectologistas. Estes últimos descrevem esta forma de alteração eletrocardiográfica para a halofantrina, que é um antimalárico utilizado até o momento unicamente para o tratamento da malária ${ }^{(9)}$.

O presente estudo tem por finalidade verificar a influência do uso de antimaláricos em pacientes reumáticos, sobre o intervalo QT.

\section{PACIENTES E MÉTODOS}

Este estudo foi realizado no Ambulatório de Reumatologia do Hospital Universitário Evangélico de Curitiba-PR. Após obtenção de consentimento informado, foram estudados 46 usuários de antimaláricos, dos quais 42 faziam uso de cloroquina e 4 de hidroxicloroquina. As doenças para as quais estes medicamentos haviam sido indicados eram: lúpus eritematoso sistêmico em 28 pacientes, artrite reumatóide em 8 pacientes, lúpus discóide em 1 paciente, osteoartrite em 8 e síndrome de Sjögren em 1 paciente. Todos os pacientes faziam uso destes medicamentos na dose de $4 \mathrm{mg} / \mathrm{kg} /$ dia para a cloroquina e $7 \mathrm{mg} / \mathrm{kg} /$ dia para a hidroxicloroquina, com exceção dos portadores de osteoartrite, que usavam a metade destes valores. A idade dos pacientes variava entre 19 e 83 anos (média de 45,35anos $\pm 15,64)$ e estes faziam uso desta droga de 1 a 84 meses (média de 27,24 meses \pm 19,76). Com exceção de dois pacientes, todos eram do sexo feminino. Os pacientes foram submetidos à realização de eletrocardiograma para medida do intervalo QTc (ou intervalo QT corrigido para a freqüência cardíaca), sendo considerado o valor normal máximo de 0,440 segundo para mulheres e 0,424 para homens ${ }^{(10)}$. O intervalo QTc foi medido manualmente com auxílio de uma lupa e por um único observador, sendo corrigido para a freqüência cardíaca pela fórmula $\mathrm{QTc}=$ QT medido/ $\sqrt{ } \mathrm{R}-\mathrm{R}^{\prime}{ }^{(8)}$.

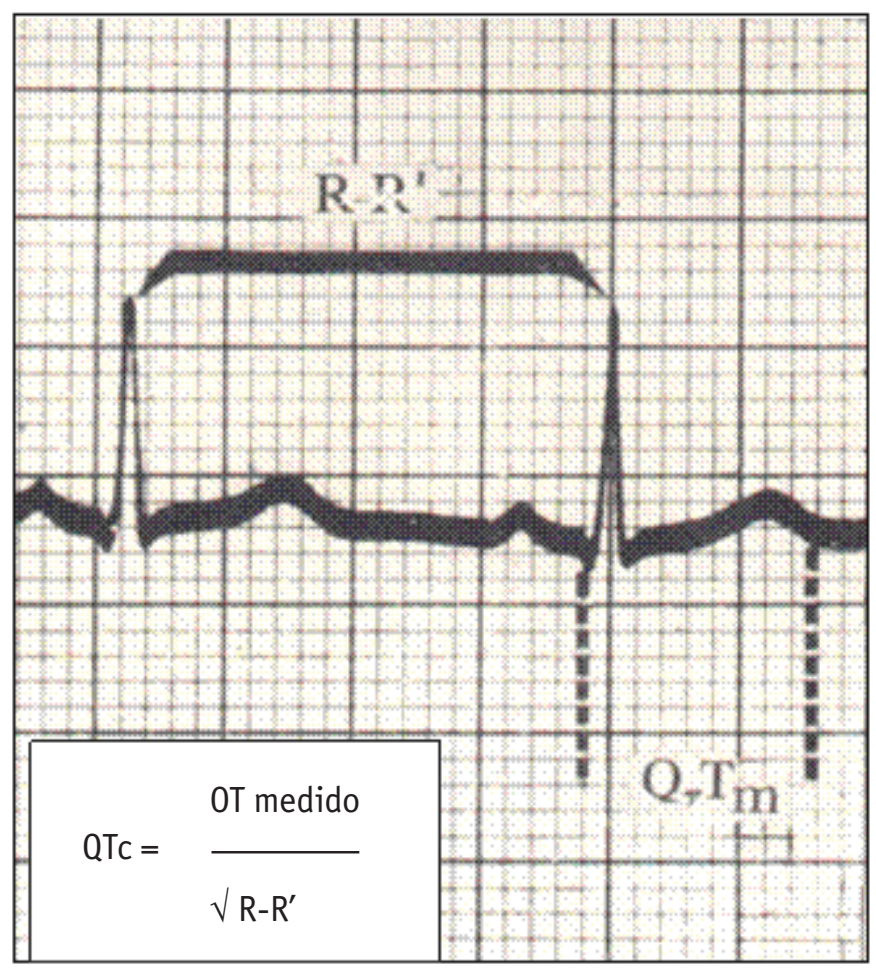

FigURA 1 - Medida do intervalo QTc (intervalo QT corrigido para a freqüência cardíaca) do eletrocardiograma. $R-R^{\prime}=$ intervalo entre duas ondas $R$ consecutivas.

Os pacientes que possuíam intervalos alterados foram solicitados a descontinuar o medicamento e novo eletrocardiograma foi realizado dentro de duas semanas para uma nova medida do intervalo QTc. 
Para estudo estatístico, foram construídas tabelas de frequiência e calculadas medidas estatísticas para descrição de conjunto de dados. Para teste de hipótese, construíram-se tabelas de contingência e realizou-se o teste exato de Fisher. O nível de significância adotado foi de 5\%.

\section{RESULTADOS}

Dos 46 pacientes estudados, 8 tinham intervalos QTc acima do normal, o que significa uma prevalência de 17,39\%. Todos os indivíduos com eletrocardiograma alterado eram usuários de cloroquina e do sexo feminino. Entretanto, em virtude das características da amostra (com poucos usuários de hidroxicloroquina), não foi possível encontrar diferença estatisticamente significativa entre os grupos de diferentes antimaláricos $(\mathrm{p}=0,452)$.

Procurando-se correlacionar a presença de alteração eletrocardiográfica com o uso do medicamento em dose completa ou pela metade, verificou-se que não existia correlação com a dose usada $(\mathrm{p}=0,574)$. Relacionando-se, ainda, as alterações eletrocardiográficas com o tempo de uso do medicamento também não foi possível encontrar correlação na amostra estudada $(p=0,09)$. Pacientes mais jovens (menos de 30 anos) tinham uma maior incidência de alongamento do intervalo QTc ( $\mathrm{p}=0,0359)$.

Dos 8 pacientes com alterações eletrocardiográficas, todos foram aconselhados a suspender o medicamento. Sete deles concordaram em repetir o eletrocardiograma após a suspensão da droga, tendo sido verificada a reversão da alteração. $\mathrm{Na}$ tabela abaixo, encontra-se uma listagem dos pacientes com alteração dos QTcs mostrando o valor antes e depois da suspensão do medicamento.

\section{DISCUSSÃO}

Entre as alterações cardíacas da cloroquina, está a cardiomiopatia vacuolar causada pelo acúmulo desta droga em lisossomos de miócitos e que aparece em menos do que $1 \%$ dos $\operatorname{casos}^{(11)}$. Bloqueios de ramo também têm sido descritos esparsamente na literatura por meio de raros relatos de casos e acredita-se que sejam resultantes da própria cardiomiopatia, a qual afeta preferentemente o septo interventricular ${ }^{(1)}$. Além disso, casos de intoxicação, em geral com intenções suicidas, sabidamente causam choque cardiogênico e parada cardíaca pelos efeitos inotrópicos negativos deste grupo de medicamentos ${ }^{(7,12)}$. Alterações no intervalo QTc pela cloroquina têm sido desencadeadas experimentalmente em miócitos ventriculares de felino estudados in vitro ${ }^{(13)}$. Como esta é uma alteração assintomática e a medida deste intervalo nem sempre é feita de rotina, ela pode passar facilmente despercebida na prática diária. Entretanto, é de suma importância. Pacientes com intervalo QT longo têm uma maior incidência de taquicardias ventriculares do tipo torsade de points, as quais podem causar síncope e até morte súbita ${ }^{(14)}$.

No presente estudo encontramos uma prevalência $17,39 \%$ de aumento no intervalo QTc nestes pacientes, os quais eram assintomáticos do ponto de vista cardíaco. Como nenhum dos indivíduos que apresentaram prolongamento do intervalo QT fazia uso de outro medicamento que pudesse ser implicado nesta anomalia e o fato de a alteração reverter nos pacientes que retiraram a droga, pode-se implicar o antimalárico diretamente na etiologia do processo.

TABELA 1

INTERVALOS QTC ANTES E DEPOIS DA SUSPENSÃO DA CLOROQUINA

\begin{tabular}{lccccc}
\hline \multirow{2}{*}{$\begin{array}{c}\text { Paciente } \\
\text { (idade em anos) }\end{array}$} & $\begin{array}{c}\text { Indicação } \\
\text { de uso }\end{array}$ & $\begin{array}{c}\text { Tempo de uso do } \\
\text { medicamento (em meses) }\end{array}$ & Dose & \multicolumn{2}{c}{ Intervalo QTC em segundos } \\
\hline 77 & OA & 04 & $1 / 2$ da dose & 0,463 & Sem medicamento \\
\hline 44 & AR & 29 & dose completa & 0,474 & 0,431 \\
62 & AR & 32 & dose completa & 0,447 & 0,431 \\
29 & LES & 43 & dose completa & 0,448 & 0,319 \\
38 & LES & 6 & dose completa & 0,446 & 0,406 \\
30 & LES & 50 & dose completa & 0,472 & 0,395 \\
30 & LES & 26 & dose completa & 0,452 & 0,407 \\
\hline 30 & LES & 38 & dose completa & 0,443 & não repetiu o ECG \\
\hline AR
\end{tabular}

$\mathrm{AR}=$ artrite reumatóide; $\mathrm{LES}=$ lúpus eritematoso sistêmico; $O \mathrm{~A}$ = osteoartrite 
Neste estudo, as alterações verificadas não puderam ser consideradas dependentes de dose ou de tempo de uso, o que sugere que o seu mecanismo possa ser similar ao da quinidina, o qual é idiossincrático, estando relacionado com tipos de canais de sódio e potássio determinados geneticamente ${ }^{(8)}$.

\section{REFERÊNCIAS}

1. Rynes RI. Antimalarial drugs. In: Keeleyu WN, Ruddy S, Harris Jr. ED, Sledge CB, editors. Textbook of Rheumatology. 5th ed. Philadelphia: WB Saunders; 1997.

2. Wallace DJ. The use of chloroquine and hydroxychloroquine for non infectious conditions other than rheumatoid arthritis or lupus: a critical review. Lupus 1996;5(Suppl 1):859-64.

3. Van Beek MJ, Piette W. Antimalarials. Dermatologic Clinics 2001;19(1):147-60.

4. Borst-Rouss M. Complete heart block as a rare complication of treatment with chloroquine. J Rheumatol 1999;26(6):1384-5.

5. Guedira N, Hajjaj-Hassouni N, Srairi JE, Fellat R, Benomar M. Third degree atrioventricular block in patient under chloroquine therapy. Rev Rheum Engl Ed 1998;65(1):58-62.

6. Iglesias Cubero G, Rodriguez Reguero JJ, Rojo Ortega JM. Restrictive cardiomyopathy caused by chloroquine. Br Heart J 1993;69:451-2

7. Rattliff NB, Estes M, Myles JL, Shirey EK, McMahon JT. Diagnosis of chloroquine cardiomyopathy by endomyocardial biopsy. N Eng J Med 1987;316(4):191-3.
Os autores sugerem que todo paciente em uso de antimalárico seja submetido a controle eletrocardiográfico com medida do intervalo QTc, que, por ser de baixo custo e de fácil acesso, pode evitar as sérias complicações desta anormalidade. Maiores estudos são necessários principalmente para verificar o papel da hidroxicloroquina neste contexto.

8. Vicent M. Long QT syndrome. Cardiology Clinics 2000; 18(2):309-25

9. Wesche DL, Schuster BG, Wang WX, Woosley RL. Mechanism of cardiotoxicity of halofantrine. Clin Pharmacol Ther 2000; 67(5):521-9.

10. Decourt LV. Considerações sobre o intervalo QT: limites superiores da normalidade. J Bras Med 1966;10:21.

11. Lacy C. Chloroquine Phosphate: drug information. In: Rose B, editor. Up to date. Versão 9.2. [Capturado 2001 set]. Disponível em: URL: http://www.uptodate.com.

12. Clemessy JL, Favier C, Borron SW, Hantson PE, Vicaut E, Baud FJ. Hypokalemia related to acute chloroquine ingestion. Lancet 1995;346:877-80.

13. Sánchez-Chapula JA, Salinas-Stefanon E, Torrés-Jacomé J, Benavides-Haro DE, Navarro-Polanco RA. Blockage of currents by antimalarial drug chloroquine in feline ventricular myocites. J Pharmacol Exp Ther 2001;297(1):437-45.

14. Passman R, Kadish A. Polymorphic ventricular tachycardia, long QT syndrome and torsades de points. Med Clin North Am 2001;85(2):321-41. 